\title{
Achieving multiple rounds of nucleic acid replication in a prebiotic solvent: A solution to the product inhibition problem
}

\author{
BRYCE E CLIFTON, ADRIANA LOZOYA COLINAS, \\ MARTHA A. GROVER AND NICHOLAS V. HUD
}

Georgia Institute of Technology

Presenting Author: bclifton3@gatech.edu

The "RNA world" hypothesis posits that RNA was responsible for its own replication before the emergence of protein enzymes. ${ }^{[1]}$ Non-enzymatic template-directed synthesis of singlestranded RNA has been achieved with activated mono- and oligonucleotides serving as reaction substrates. ${ }^{[2,3]}$ For additional rounds of replication the strands of the duplex that result from a template-directed synthesis reaction must be separated so that they can function as templates for the organization of new reaction substrates. While thermal denaturation is typically used to separate the strands of an RNA duplex, upon cooling the duplex strands will reassociate before substrate binding, a quandary known as The Product Inhibition Problem. ${ }^{[3-5]} \mathrm{We}$ report that a solvent composed of the prebiotically plausible molecules urea, acetamide, and water enables multiple rounds of replication of an RNA duplex. ${ }^{[6]}$ Heat cycling within our proposed prebiotic solvent promotes the binding of substrate oligonucleotides to the template of a denatured duplex under a variety of conditions, including variations in salt concentration, solvent composition, and water activity. In addition, templatedirected substrate ligation within chemical activation results in higher yields in our solvent in comparison to water. The environmental conditions that support the existence of our solvent (i.e., temperature, relative humidity) were likely widely available on the prebiotic Earth and the variations in environmental conditions necessary for circumventing the product inhibition problem could have been driven by regular geophysical processes, such as Earth's water cycle and day-night cycles. These results demonstrate the importance of solvent properties on controlling prebiotic replication and may present environmental constraints on the conditions in which life originated.

[1] Szostak, J. W. (2012), J. Sys. Chem. 3.

[2] Adamala, K. \& Szostak, J. W. (2013), Science 342, 10981100 .

[3] von Kiedrowski, G. (1986), Angew. Chem. Int. Ed. Engl. $25,932-935$

[4] He, C., Gallego, I., Laughlin, B., Grover, M. A., \& Hud, N. V. (2017), Nat. Chem. 9, 318-324.

[5] He, C., Lozoya, A., Gallego, I., Grover, M. A., \& Hud, N. V. (2019), Nucleic Acids Res. 47, 6569-6577.

[6] Miller, S., Urey, H., \& Oró, J. (1976), J. Mol. Evol. 9, 5972. 